\title{
Evaluation of the efficacy and safety of hydroxychloroquine in comparison with chloroquine in moderate and severe patients with COVID-19
}

\author{
Qiuyu $\mathrm{Li}^{1 \dagger}$, Cheng $\mathrm{Cui}^{2 \dagger}$, Fei Xu ${ }^{3 \dagger}$, Jianping Zhao ${ }^{4}$, Nan $\mathrm{Li}^{5}$, Haichao $\mathrm{Li}^{6}$, Tianbing Wang ${ }^{7}$, \\ Hua Zhang ${ }^{5}, \mathrm{Na} \mathrm{Liu}^{2,8}$, Yudong $\mathrm{Wei}^{2}$, Xiaoye $\mathrm{Niu}^{2}$, Yan $\mathrm{Xu}^{2}$, Jianping Dong ${ }^{8}$, Xueting Yao , \\ Xiaohong Wang ${ }^{1}$, Yahong Chen ${ }^{1}$, Haiyan $\mathrm{Li}^{2,9}$, Chunli Song ${ }^{10}$, Jie Qiao ${ }^{10}$, Dongyang $\mathrm{Liu}^{2 *} \&$ \\ Ning Shen ${ }^{1 *}$ \\ ${ }^{1}$ Department of Respiratory and Critical Care Medicine, Peking University Third Hospital, Beijing 100191, China; \\ ${ }^{2}$ Drug Clinical Trial Center, Peking University Third Hospital, Beijing 100191, China; \\ ${ }^{3}$ Department of Respiratory and Critical Care Medicine, the First Affiliated Hospital of Nanchang University, Nanchang 330006, China; \\ ${ }^{4}$ Department of Respiratory and Critical Care Medicine, Tongji Hospital, Tongji Medical College, Huazhong University of Science and \\ Technology, Wuhan 430030, China; \\ ${ }^{5}$ Research Center of Clinical Epidemiology, Peking University Third Hospital, Beijing 100191, China; \\ ${ }^{6}$ Department of Respiratory and Critical Care Medicine, Peking University First Hospital, Beijing 100034, China; \\ ${ }^{7}$ Trauma Center, Peking University People's Hospital, Beijing 100044, China; \\ ${ }^{8}$ Beijing Haidian Hospital, Haidian Section of Peking University Third Hospital, Beijing 100080, China; \\ ${ }^{9}$ Department of Cardiology, Peking University Third Hospital, Beijing 100191, China; \\ ${ }^{10}$ Institute of Medical Innovation, Peking University Third Hospital, Beijing 100191, China
}

Received August 19, 2020; accepted December 18, 2020; published online January 18, 2021

Citation: Li, Q., Cui, C., Xu, F., Zhao, J., Li, N., Li, H., Wang, T., Zhang, H., Liu, N., Wei, Y., et al. (2021). Evaluation of the efficacy and safety of hydroxychloroquine in comparison with chloroquine in moderate and severe patients with COVID-19. Sci China Life Sci 64, 660-663. https://doi.org/ $10.1007 / \mathrm{s} 11427-020-1871-4$

Dear Editor,

Since December 2019, severe acute respiratory syndrome coronavirus 2 (SARS-CoV-2) has been rapidly spreading worldwide. Hydroxychloroquine and chloroquine are candidates for the treatment of coronavirus disease 2019 (COVID-19) owing to its antiviral effect and immunomodulation properties. However, the published results of utilizing hydroxychloroquine and chloroquine as treatment were still controversial and their efficacy and safety in patients with COVID-19 need to be further elucidated.

A total of 88 laboratory-confirmed (identified by viral

$\dagger$ Contributed equally to this work

*Corresponding authors (Ning Shen, email: shenning1972@126.com; Dongyang Liu, email: liudongyang@vip.sina.com)
RNA or antibody tests) patients with COVID-19 (65 moderate and 23 severe patients with COVID-19) were included in our multicenter, randomized controlled trial (RCT). Eligible patients randomly assigned in a 1:1 ratio to orally receive hydroxychloroquine $(600 \mathrm{mg}$ twice a day on the first day followed by $200 \mathrm{mg}$ twice a day in the last four days) plus standard care or chloroquine $(500 \mathrm{mg}$ twice a day for the first three days followed by $250 \mathrm{mg}$ twice a day in the last two days) plus standard care (SC) for 5 days. Our study protocol is provided in the Supplementary Methods. The average age of our patients was 61.3 years old and they had a higher risk of developing severe illness. The average time interval between the onset of symptoms and hospitalization was 17.6 days for the patients in our study, which was much longer than the previously published statistics, indicating a 
relatively late stage of COVID-19 (Li et al., 2020; Zhou et al., 2020). The duration between hospitalization and randomization was roughly 10 days, wherein most of the patients $(67 \%-81 \%)$ underwent a series of medical treatments that did not result in improvement. The details of basic demographics and clinical status are presented in Table S1 (Supporting Information). The factors that make the patients in our study a special subgroup of patients with COVID-19 include old age, slow disease progression, and poor response to multiple antiviral medications. Currently, there are no reports on the efficacy of hydroxychloroquine and chloroquine in such patients.

By analyzing our prespecified primary outcomes, we found that there was no statistical difference between the hydroxychloroquine and chloroquine groups in terms of the time to clinical recovery (TTCR) in moderate patients and the time to clinical improvement (TTCI) in severe patients. The median of TTCR in moderate patients was 9 days in both the hydroxychloroquine and chloroquine treated groups (HR, $0.54 ; 95 \% \mathrm{CI}, 0.17$ to $1.73 ; P=0.30$ ). In the severe cases, the median of TTCI was 13 days in the hydroxychloroquine group and 18 days in the chloroquine group (HR, 0.78; 95\% CI, 0.26 to $2.35 ; P=0.66$ ) (Figure $1 \mathrm{~A}$ and $\mathrm{B}$ ). We also analyzed the length of hospital stay (LOS) to better understand the overall recovery time. In the moderate cases, the median LOS was 11 days in the hydroxychloroquine group and 13 days in the chloroquine group. For the severe cases of COVID-19, the median LOS was 20 days in the hydroxychloroquine group and 19 days in the chloroquine group (HR, 0.63; 95\% CI, 0.18 to $2.24 ; P=0.47$ ) (Figure $1 \mathrm{C}$ and D).

In our study, we observed that the viral load substantially decreased after the hydroxychloroquine and chloroquine treatment. SARS-CoV-2 was only detected in $13.0 \%(N=3)$ of moderate cases in the hydroxychloroquine group and $25.9 \%(N=7)$ of moderate cases in the chloroquine group (difference, $-12.88 ; 95 \% \mathrm{CI},-34.39,8.63 ; P=0.26$ ). All severe cases in the hydroxychloroquine group tested negative in the viral RNA test, whereas $14.3 \%(N=1)$ of the severe cases in the chloroquine group tested positive on day 5 (difference, $-14.29 ; 95 \% \mathrm{CI},-40.21$ to $11.64 ; P=0.27$ ). The results showing that chloroquine could accelerate achieving undetectable viral RNA are consistent with the study by Huang et al. (2020). However, the RCT results published by Wei Tang demonstrated that hydroxychloroquine did not show significant improvement in the probability of negative conversion in mild to moderate COVID-19 cases (Tang et al., 2020). Inappropriate stratification of COVID-19 patients, lack of proper dose optimization, and delayed administration against the self-limited virus may have contributed to these inconclusive results.

Previous research showed that SARS-CoV-2 attacks Tcells, and the decline of T-cells was closely related to the increase in inflammatory cytokines (Liu et al., 2020). An increase in proinflammatory cytokines can trigger a cytokine storm, which is one of the critical risks of the progression to severe illness (Huang et al., 2020; Moon, 2020; Qin et al., 2020; Vaninov, 2020). Therefore, maintaining T-cell function and reducing the risks of developing a cytokine storm is crucial for COVID-19 patients. Hydroxychloroquine has been found to alleviate the decline of T-cell counts and reduce the overactivation of B cells (Piconi et al., 2011; Torigoe et al., 2018). The observation in our study suggests that patients in the hydroxychloroquine group experience a better improvement in the response of lymphocytes and ferritin compared to the chloroquine group (Laboratory outcomes are fully listed in Tables S2 and S3, and other secondary outcomes are in Table S4 in Supporting Information). Considering the characteristics of COVID-19 and the key role of the immune system in infectious diseases, future studies on hydroxychloroquine and chloroquine should focus on its immunomodulatory effect.

Chest computed tomography (CT) could provide valuable information on the prognostication of COVID-19. We also examined the $\mathrm{CT}$ results by $\mathrm{CT}$ scores and found that the $\mathrm{CT}$ scores of the hydroxychloroquine group in severe cases were significantly improved compared with the chloroquine group. Alternatively, there was no significant difference in moderate cases. The changes from the baseline CT scores in the moderate cases were 0.81 and 1.75 in the hydroxychloroquine and the chloroquine group, respectively (difference, $-0.94 ; 95 \% \mathrm{CI},-2.12$ to $1.01 ; P=0.46$ ). The change from the baseline CT score in severe cases in the hydroxychloroquine group was 3.63, compared to 0.67 in the chloroquine group (difference, 2.96; 95\% CI, 2.28 to 8.35 ; $P<0.01)$.

Furthermore, we also introduced a dataset from a realworld study (RWS), which were collected from the patients in the same hospital, to compare with the RCT results to determine the efficacy of the hydroxychloroquine/chloroquine (Baseline characteristics of the matched RCT and RWS patients are presented in Table S5 in Supporting Information). There was no significant statistical difference between RCT and RWS groups in the duration of symptom onset to discharge (Figure S1 in Supporting Information), CT scores, and percentages of patients with detectable viral RNA. However, the level of CRP was substantially decreased in the RCT group. After baseline correction, the change of CRP from the baseline in the RCT group was significantly different ( 30.50 vs. 5.09 , difference $5.90,95 \%$ CI, 0.20 to $11.60, P=0.04$ ) from the RWS group on day 10 (Table S6 in Supporting Information). Recently, several studies showed that coagulation dysfunction, characterized by increased D-dimer levels and decreased platelet counts, was one of the major complications of COVID-19 (Guan et al., 2020; Huang et al., 2020; Tang et al., 2020). Despite the missing data in the RWS population, we are pleased to fully 

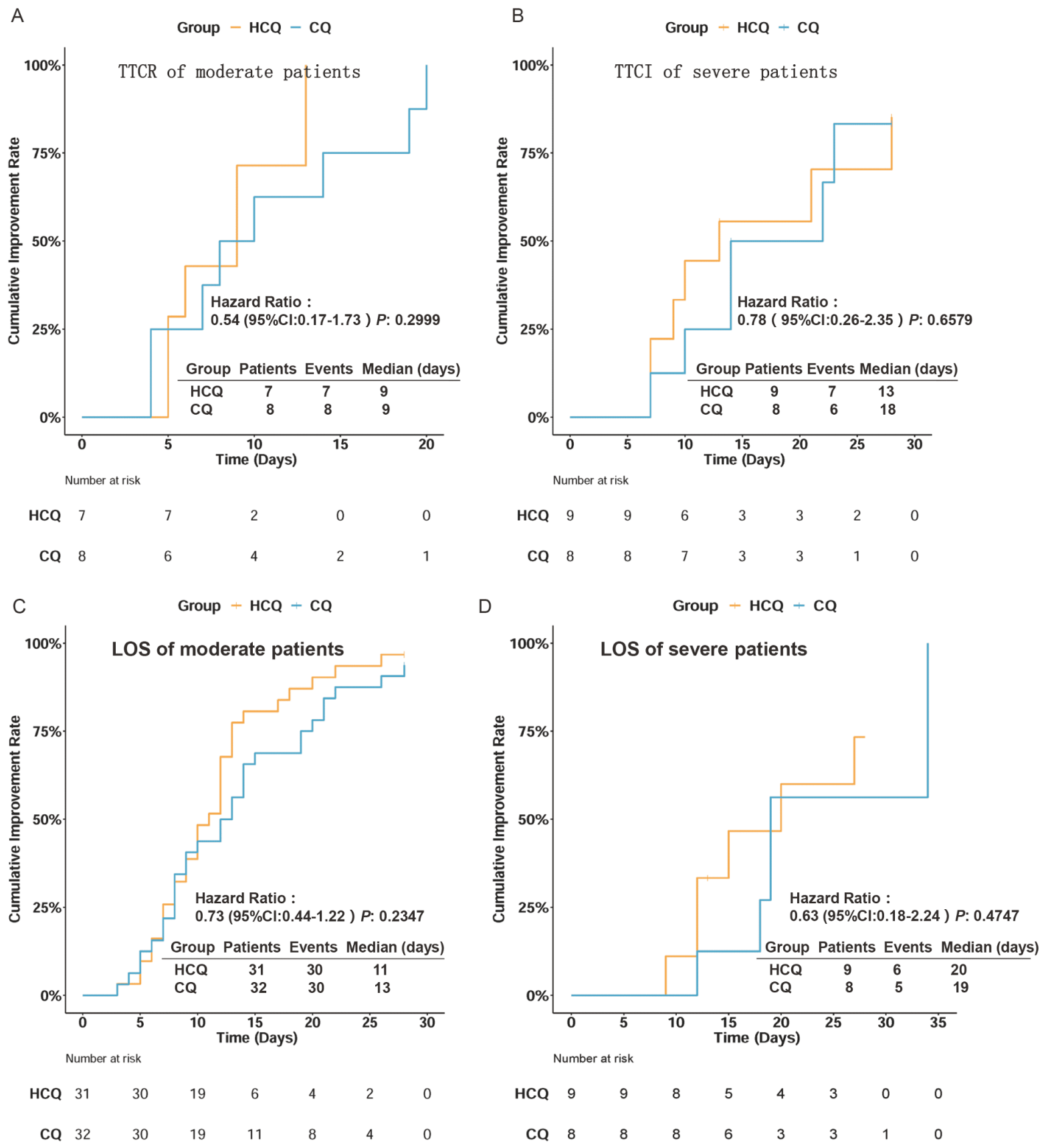

Figure 1 Primary outcomes and length of hospital stay (LOS) in the moderate and severe COVID-19 cases. A, Time to clinical recovery (TTCR) in moderate cases. B, Time to clinical improvement (TTCI) in severe cases. C, LOS of moderate cases. D, LOS of severe cases.

disclose the data of coagulation-related parameters of RCT and RWS in Table S7 in Supporting Information.

In addition, the clinical safety profiles of hydroxychloroquine/chloroquine are well-known in patients affected by malaria and rheumatoid arthritis, and the adverse events are generally transient and manageable with the appropriate administration of the drug. In our study, we also found that hydroxychloroquine and chloroquine were well tolerated in patients with COVID-19. In moderate cases, 29.03\% (9/31) of the patients in the hydroxychloroquine group and 54.55\% $(18 / 33)$ of the patients in the chloroquine group reported adverse events during the trial. For the severe cases, $36.36 \%$ $(4 / 11)$ of the patients in the hydroxychloroquine group and $40.00 \%(4 / 10)$ of the patients in the chloroquine group reported adverse events during the trial. The most common drug-related adverse events (DRAEs) were diarrhea and QT prolongation. All DRAEs were grade 1 and patients could spontaneously recover from these events (Table S8 in Sup- 
porting Information).

Several limitations exist in our study. First, our clinical trials were terminated earlier than expected. The number of new cases of COVID-19 in Hubei province dramatically decreased on March 18. We failed to include enough patients in our study. The consequence of an early termination was the reduced sample size, which could further impact the power of our analysis. Second, the patients' outcomes in our study may not be enough representation of all patients with COVID-19. Patients who have access to early diagnosis and early intervention may have better clinical outcomes according to the antiviral characteristics of other drugs.

In conclusion, the primary clinical outcomes (TTCR and TTCI) of hydroxychloroquine treatment were not significantly different from the chloroquine treatment in the 28day trial period in patients with persistent moderate to severe COVID-19 symptoms. Hydroxychloroquine may exert a more promising efficacy in immune system modulation, indicated by ferritin reduction in the moderate cases and improvement of CT scores and lymphocyte counts in the severe cases. Hydroxychloroquine and chloroquine are well tolerated in our patients. Furthermore, carefully designed studies are needed to fully establish the exact clinical efficacy and safety of treatment with hydroxychloroquine monotherapy or combination treatment with other antiviral candidate drugs in patients with COVID-19.

Trial registration: Chinese Clinical Trial Register number: ChiCTR2000029898, ChiCTR2000029899, ChiCTR2000031376.

Compliance and ethics The author(s) declare that they have no conflict of interest.

Acknowledgements We thank the trial team members at Peking University First Hospital, Peking University People's Hospital, the First Affiliated Hospital of Nanchang University and Tongji Hospital, Tongji Medical College of Huazhong University of Science and Technology. We thank Dr. Yongchang Sun, Dr. Jinxia Zhao, Dr. Hongmei Jing, Dr. Xiaoguang Li, Dr Yahong Wang, Dr. Qinggang Ge, Dr. Chen Yao, Dr. Xiaoyan Yan, Dr. Wenle Zhao, Dr. Naiqing Zhao, Dr. Cheng Lv, Dr. Zijian Hu, Dr. Chang Liu and Dr. Lei Wang for the scientific discussion. We thank Shanghai Pharmaceutical Co., Ltd. and Guangdong ZhongSheng Pharmaceutical Co., Ltd. for providing the research drug. We thank all the members of the drug clinical trial center of Peking University Third Hospital for the whole project. This work was supported by MOST (Ministry of Science and Technology of the People's Republic of China) foundation for SARS-CoV-2 Research (2020YFC0844500), the "13th Five-Year" National Science and Technology Major Project of China (2017ZX09101001-002-001, 2017ZX09304012), and Peking University Health Center Foundation for Combating the Pandemic Programs (BMU2020HKYZX011).

\section{References}

Guan, W.J., Ni, Z.Y., Hu, Y., Liang, W.H., Ou, C.Q., He, J.X., Liu, L., Shan, H., Lei, C.L., Hui, D.S.C., et al. (2020). Clinical characteristics of coronavirus disease 2019 in China. N Engl J Med 382, 1708-1720.

Huang, C., Wang, Y., Li, X., Ren, L., Zhao, J., Hu, Y., Zhang, L., Fan, G., $\mathrm{Xu}, \mathrm{J} ., \mathrm{Gu}, \mathrm{X}$., et al. (2020). Clinical features of patients infected with 2019 novel coronavirus in Wuhan, China. Lancet 395, 497-506.

Li, Q., Guan, X., Wu, P., Wang, X., Zhou, L., Tong, Y., Ren, R., Leung, K. S.M., Lau, E.H.Y., Wong, J.Y., et al. (2020). Early transmission dynamics in Wuhan, China, of novel coronavirus-infected pneumonia. N Engl J Med 382, 1199-1207.

Liu, J., Li, S., Liu, J., Liang, B., Wang, X., Wang, H., Li, W., Tong, Q., Yi, J., Zhao, L., et al. (2020). Longitudinal characteristics of lymphocyte responses and cytokine profiles in the peripheral blood of SARS-CoV-2 infected patients. EBioMedicine 55, 102763.

Moon, C. (2020). Fighting COVID-19 exhausts T cells. Nat Rev Immunol 20, 277.

Piconi, S., Parisotto, S., Rizzardini, G., Passerini, S., Terzi, R., Argenteri, B., Meraviglia, P., Capetti, A., Biasin, M., Trabattoni, D., et al. (2011). Hydroxychloroquine drastically reduces immune activation in HIVinfected, antiretroviral therapy-treated immunologic nonresponders. Blood 118, 3263-3272.

Qin, C., Zhou, L., Hu, Z., Zhang, S., Yang, S., Tao, Y., Xie, C., Ma, K., Shang, K., Wang, W., et al. (2020). Dysregulation of immune response in patients with coronavirus 2019 (COVID-19) in Wuhan, China. Clin Infect Dis 71, 762-768.

Tang, N., Li, D., Wang, X., and Sun, Z. (2020). Abnormal coagulation parameters are associated with poor prognosis in patients with novel coronavirus pneumonia. J Thromb Haemost 18, 844-847.

Tang, W., Cao, Z., Han, M., Wang, Z., Chen, J., Sun, W., Wu, Y., Xiao, W., Liu, S., Chen, E., et al. (2020). Hydroxychloroquine in patients with mainly mild to moderate coronavirus disease 2019: open label, randomised controlled trial. BMJ 369, m1849.

Torigoe, M., Sakata, K., Ishii, A., Iwata, S., Nakayamada, S., and Tanaka, Y. (2018). Hydroxychloroquine efficiently suppresses inflammatory responses of human class-switched memory $B$ cells via Toll-like receptor 9 inhibition. Clin Immunol 195, 1-7.

Vaninov, N. (2020). In the eye of the COVID-19 cytokine storm. Nat Rev Immunol 20, 277.

Zhou, F., Yu, T., Du, R., Fan, G., Liu, Y., Liu, Z., Xiang, J., Wang, Y., Song, B., Gu, X., et al. (2020). Clinical course and risk factors for mortality of adult inpatients with COVID-19 in Wuhan, China: a retrospective cohort study. Lancet 395, 1054-1062.

\section{SUPPORTING INFORMATION}

The supporting information is available online at https://doi.org/10.1007/s11427-020-1871-4. The supporting materials are published as submitted, without typesetting or editing. The responsibility for scientific accuracy and content remains entirely with the authors. 